\title{
Numerical study of nonlinear shallow water waves produced by a submerged moving disturbance in viscous flow
}

\author{
Daohua Zhang and Allen T. Chwang \\ Department of Mechanical Engineering, The University of Hong Kong, Pokfulam Road, Hong Kong
}

(Received 13 March 1995; accepted 25 September 1995)

\begin{abstract}
Two-dimensional solitary waves generated by a submerged body moving near the critical speed in a shallow water channel are studied numerically. The incompressible Navier-Stokes equations in a curvilinear free-surface-fitted coordinate system are solved by the finite difference method. The present numerical results are compared with the existing experimental data, and with the numerical solutions of two inviscid-flow models, i.e. the general Boussinesq equation and the forced Korteweg-de Vries equation. It is found that the viscous effect in the boundary layer around the body and on the bottom of the channel plays an important role in the generation of solitary waves on the free surface. Hence the Navier-Stokes solutions have a better agreement with the experimental data than those obtained from two inviscid-flow models. The effect of the submergence depth of the body on the waves generated is also investigated. It reveals that waves are insensitive to the submergence depth of the body, except for a small region quite close to the bottom of the water channel. (C) 1996 American Institute of Physics. [S1070-6631(96)02901-2]
\end{abstract}

\section{INTRODUCTION}

A disturbance moving with a transcritical velocity in shallow water can periodically generate a succession of solitary waves advancing upstream of the disturbance. Immediately behind the moving disturbance there trails an ever elongating stretch of a uniformly depressed water surface followed by a train of cnoidal-like waves oscillating about the initial free-surface level, decreasing in amplitude with distance and eventually vanishing in the farfield. The disturbance can be a surface pressure distribution, a submerged body, or a bottom topography. Although the disturbance moves at a constant speed (steady motion), the flow generated can be unsteady and periodic. The first recorded observation of this phenomenon was reported, as a "great solitary wave," by John Scott Russell in 1834. A century later, this phenomenon was observed by Thews and Landweber ${ }^{1}$ in a series of towing tank tests of ship models in shallow water. Thereafter several systematic experimental investigations were reported. ${ }^{2-4}$

This phenomenon was rediscovered, first numerically by $\mathrm{Wu}$ and $\mathrm{Wu}^{5}$ based on the generalized Boussinesq ( $\mathrm{g}-\mathrm{B}$ ) model derived earlier by $\mathrm{Wu}^{6}$ for describing two-dimensional long waves with moving surface pressure or bottom topography. A simplified forced Korteweg-de Vries (fKdV) model derived from the Boussinesq equation was employed by $\mathrm{Wu}^{7}$ to explore the basic mechanism underlying the phenomenon. Lee et $a l^{8}{ }^{8}$ carried out a combined numerical and experimental study with a two-dimensional cambered bottom topography moving along the bottom of a water channel in order to verify the numerical solutions of theoretical models, i.e., the g-B model and the fKdV model. A broad agreement between the experimental data and solutions derived from two theoretical models were found, in terms of both the amplitude and phase of the generated waves.

In addition to these studies there were papers reporting calculations using the Korteweg-de Vries (KdV) equation by Akylas $^{9}$ and Cole, ${ }^{10}$ and using the $\mathrm{fKdV}$ model by Grimshaw and Smyth. ${ }^{11}$ These studies have also been extended to nonlinear waves in two horizontal dimensions by $\mathrm{Mei}^{12}$ based on approximations for slender bodies in a shallow channel, by Ertekin et al. ${ }^{13}$ employing Green-Nagdi's theory, by Wu and $\mathrm{Wu}^{14}$ based on the g-B model, and by Katsis and Akylas ${ }^{15}$ using the Kadomtsev-Petviashvili model.

Besides the simplified theoretical studies, many numerical schemes for two-dimensional potential flows with fully nonlinear boundary conditions on the free surface have been presented. Longuet-Higgins and Cokelet ${ }^{16}$ applied the boundary element method based on the Green's integral theorem with the mixed Eulerian-Lagrangian scheme to the simulation of transient free-surface flows. Their work was modified by Suzuki $^{17}$ for the problem of a semicircular mound in shallow water and by Cointe ${ }^{18}$ for the shallowwater swell. For problems of wave-body interactions, Yeung et al. ${ }^{19}$ developed a finite difference method to solve the Laplace equation using a mixed Eulerian-Lagrangian formulation to satisfy the fully nonlinear free-surface conditions. $\mathrm{Cao}$ and $\mathrm{Beck}^{20}$ presented a simple numerical method using the complete Laplace equation and fully nonlinear freesurface boundary conditions to include the higher-order effect. The method was applied to calculate two-dimensional solitary waves generated by a moving surface pressure disturbance, a submerged circular cylinder, and a bottom bump. From the numerical results, it was found that for strong disturbances, the fully nonlinear model predicted larger waves than those predicted by the fKdV model. The fully nonlinear calculations showed that a free-surface pressure distribution generated significantly larger waves than those due to a bottom topography.

The theoretical and numerical studies of this phenomenon mentioned above are based on the potential-flow theory. The main limitation of these methods is that the viscous effect cannot be accounted for, except in a very crude way for the modeling of the bottom friction. To analyze the complexities of the phenomenon, including nonlinear effects, dispersion, and dissipation, a more general formulation is re- 
quired. Solutions of Navier-Stokes (NS) equations with consistent viscous boundary conditions offer an opportunity to capture these complexities. The earliest and most famous method for solving time-dependent viscous flows with a free surface is the MAC method by Harlow and Welch. ${ }^{21}$ This method has been improved and extended to many different versions, for instance the SUMAC method of Chan and Street, ${ }^{22}$ the TUMAC method of Miyata et al. ${ }^{23}$ and the method of Tang et al. ${ }^{24}$ Because the MAC-type methods use a spatial fixed Cartesian coordinate system, they require special treatment on the boundaries of arbitrary shape such as the deformed free surface and body boundary. Therefore these methods are not particularly well suited for the freesurface flow around a body with a complicated geometry. On the other hand, a method based on a free-surface-fitted coordinate system has been considered as an alternative approach to solve the fully nonlinear free-surface problem. Methods of this type have been developed and applied to a variety of nonlinear wave problems. ${ }^{25-28}$ Chang and Tang $^{26}$ studied nonlinear water waves generated by a bottom bump, and found that the amplitudes of advancing solitary waves and downstream train waves were attenuated by the viscous effect at low Reynolds numbers. Hinatsu ${ }^{27}$ investigated various nonlinear wave problems, including the wave generated by a moving topography.

In the present work, numerical solutions of the NS equations in primitive variables using a moving grid system fitted to the free surface are solved to simulate nonlinear solitary waves generated by a submerged body moving along the horizontal bottom of a water channel. A finite difference method similar to the scheme of Hinatsu ${ }^{27}$ is adopted for the present computation. As mentioned before, most theoretical and numerical studies of nonlinear solitary waves generated by a submerged body were based on the inviscid-flow theory. Although a few attempts were made in these studies to compare theories with experiments, only qualitative agreement between them can be inferred. Lee et al. ${ }^{8}$ made a quantitative comparison between their experimental data and the numerical results of the fKdV and g-B models, in which the viscous effects were accounted for in a crude way, and found reasonably good agreement. The results of the present viscous flow analysis with consistent viscous boundary conditions are compared with the experimental data and the numerical results of the g-B model and the fKdV model obtained by Lee et al. ${ }^{8}$ for validating the present computational method. A series of calculations are conducted for various submergence depths of the body in order to investigate the effect of the submergence depth of the body on the generated waves.

\section{GOVERNING EQUATIONS}

The nonlinear wave problem considered in the present paper is shown in Fig. 1. A Cartesian coordinate system fixed with respect to the body is chosen with the $x_{1}$ axis along the bottom of the channel and $x_{2}$ axis pointing upward. In this body frame, the body is fixed in a uniform stream of velocity $u_{0}$ with unperturbed water depth $h_{0}$. The motion of an incompressible viscous fluid is governed by the NS equations

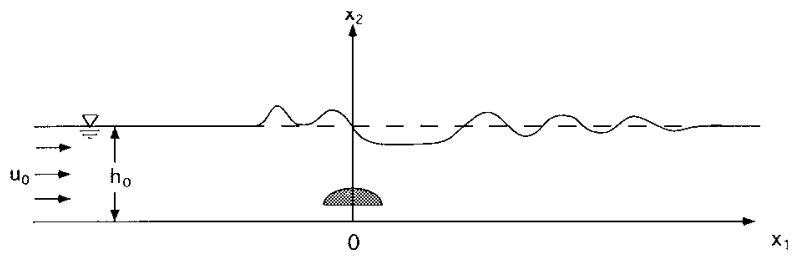

FIG. 1. A submerged disturbance moving with a transcritical velocity in shallow water.

and the continuity equation. The dimensionless conservative form of the two-dimensional NS equations in this Cartesian coordinate system is

$$
\frac{\partial u_{i}}{\partial t}+\frac{\partial\left(u_{i} u_{j}\right)}{\partial x_{j}}=-\frac{\partial p}{\partial x_{i}}+\frac{1}{\operatorname{Re}} \frac{\partial}{\partial x_{j}}\left(\frac{\partial u_{i}}{\partial x_{j}}\right)-\frac{1}{F n^{2}} \frac{\partial x_{2}}{\partial x_{i}},
$$

and the continuity equation is

$$
\frac{\partial u_{i}}{\partial x_{i}}=0,
$$

where $u_{i}$ is the velocity component along the $x_{i}$ axis, $p$ is the pressure, and $t$ is the time. The governing equations are normalized with the steady velocity of the disturbance (or in the body frame, the uniform stream velocity) $u_{0}$ and the initial uniform water depth $h_{0}$. The Reynolds number and Froude number are defined as $\operatorname{Re}=u_{0} h_{0} / \nu$ and $F n=u_{0} / \sqrt{g h_{0}}$, respectively, where $g$ is the gravitational constant and $\nu$ is the kinematic viscosity coefficient.

For accurate computation of viscous flow with a free surface, it is necessary to transform the governing equations to a time-dependent, curvilinear coordinate system fitted to the moving free surface as well as the body boundaries. A curvilinear transformation from the physical coordinate system to the computational coordinate system is defined by

$$
\xi^{j}=\xi^{j}\left(x_{i}, t\right) \text { and } \tau=\tau(t) .
$$

In the above transformation, $\tau$ is a function of $t$ only, and in the present paper it is assumed that $\tau=t$. The Jacobian of the transformation is

$$
J=\frac{\partial\left(\xi^{1}, \xi^{2}\right)}{\partial\left(x_{1}, x_{2}\right)} .
$$

Equations (1) and (2) can be partially transformed to the computational coordinate system $\left(\xi^{i}, t\right)$ as

$$
\begin{aligned}
\frac{\partial}{\partial t}\left(\frac{u_{i}}{J}\right)+\frac{\partial}{\partial \xi^{j}}\left(\frac{u_{i}}{J} \frac{\partial \xi^{j}}{\partial t}+\frac{u_{i}}{J} U^{j}\right) \\
=-\frac{\partial}{\partial \xi^{j}}\left(\frac{\phi}{J} \frac{\partial \xi^{j}}{\partial x_{i}}\right)+\frac{1}{\operatorname{Re}} \frac{\partial}{\partial \xi^{j}}\left(\frac{1}{J} g^{j l} \frac{\partial u_{i}}{\partial \xi^{l}}\right)
\end{aligned}
$$

and

$$
\frac{\partial}{\partial \xi^{j}}\left(\frac{U^{j}}{J}\right)=0
$$

with

$$
U^{j}=\frac{\partial \xi^{j}}{\partial x_{k}} u_{k}, \quad \phi=p+\frac{x_{2}}{F n^{2}}, \quad g^{j l}=\frac{\partial \xi^{j}}{\partial x_{k}} \frac{\partial \xi^{l}}{\partial x_{k}},
$$


where $U^{j}$ is the contravariant velocity component along the $\xi^{j}$ direction, $g^{j l}$ is the metric tensor, $\phi$ is the redefined pressure term, and $\partial \xi^{j} / \partial t$ associated with the grid movement is the grid point speed. The following metric identities are used for the transformation:

$$
\frac{\partial}{\partial \xi^{j}}\left(\frac{1}{J} \frac{\partial \xi^{j}}{\partial x_{i}}\right)=0, \quad \frac{\partial}{\partial t}\left(\frac{1}{J}\right)+\frac{\partial}{\partial \xi^{j}}\left(\frac{1}{J} \frac{\partial \xi^{j}}{\partial t}\right)=0 .
$$

\section{BOUNDARY CONDITIONS}

The free-surface boundary is not known a priori. The location of the free surface is part of the solution in the problem and is determined from the free-surface boundary conditions. The free-surface boundary conditions consist of one kinematic condition and two dynamic conditions. The kinematic condition states that the fluid particles of the free surface stay on the free surface at any time. If $h\left(x_{1}, t\right)$ is the free-surface elevation, this condition is

$$
\frac{\partial h}{\partial t}+u_{1} \frac{\partial h}{\partial x_{1}}=u_{2}, \quad \text { at } x_{2}=h\left(x_{1}, t\right) .
$$

The dynamic conditions represent the continuity of stresses on the free surface. Neglecting the surface tension on the free surface, the dynamic conditions are

$$
n_{i} \sigma_{i j} n_{j}=0
$$

and

$$
t_{i} \sigma_{i j} n_{j}=0, \quad \text { at } x_{2}=h\left(x_{1}, t\right),
$$

where $n_{i}$ is the unit normal vector and $t_{i}$ is the unit tangential vector to the free surface. The stress tensor for an incompressible Newtonian fluid can be expressed as

$$
\sigma_{i j}=-p \delta_{i j}+\frac{1}{\operatorname{Re}}\left(\frac{\partial u_{j}}{\partial x_{i}}+\frac{\partial u_{i}}{\partial x_{j}}\right),
$$

where $\delta_{i j}$ is the Kronecker delta. Equations (9) and (12) can be partially transformed into the curvilinear coordinate system $\left(\xi^{i}, t\right)$ as

$$
\frac{\partial h}{\partial t}+\left(\frac{\partial \xi^{i}}{\partial t}+u_{1} \frac{\partial \xi^{i}}{\partial x_{1}}\right) \frac{\partial h}{\partial \xi^{i}}=u_{2}
$$

and

$$
\sigma_{i j}=-p \delta_{i j}+\frac{1}{\operatorname{Re}}\left(\frac{\partial u_{j}}{\partial \xi^{l}} \frac{\partial \xi^{l}}{\partial x_{i}}+\frac{\partial u_{i}}{\partial \xi^{l}} \frac{\partial \xi^{l}}{\partial x_{j}}\right) .
$$

The velocity component $u_{1}$ on the free surface is extrapolated from the interior in such a way that the dynamic free-surface boundary conditions are satisfied on the free surface. The velocity gradient $\partial u_{1} / \partial \xi^{2}$ on the free surface, which is needed for the extrapolation, are obtained by solving Eqs. (10) and (11) simultaneously. The velocity component $u_{2}$ on the free surface is calculated from the continuity equation (2). The normal dynamic free-surface condition, Eq. (10), is used to specify the pressure on the free surface. And the kinematic boundary condition, Eq. (9), is used to determine the location of the free surface.

The no-slip boundary condition is imposed on the body boundary surface. In the body frame, the body is fixed, whereas the bottom of the channel moves with uniform velocity $u_{0}$. Therefore the free-slip boundary condition is used on the bottom. These conditions are stated mathematically as

$$
\begin{aligned}
& u_{1}=u_{0}, u_{2}=0, \quad \text { on the bottom, } \\
& u_{1}=0, u_{2}=0, \quad \text { on the body surface. }
\end{aligned}
$$

For the case where the body touches the bottom, Neumann (zero gradient) boundary condition, $\partial u_{1} / \partial \xi^{2}=0$, is specified on the bottom of the channel to avoid the singularity. Far upstream, flow is uniform and the free surface is undisturbed. Thus

$$
u_{1}=u_{0}, u_{2}=0, \quad \text { as } x_{1} \rightarrow-\infty .
$$

The open boundary conditions for free-surface problems are treated by various methods. ${ }^{28,29}$ To prevent the reflection of waves into the solution domain the open boundary conditions must be carefully implemented. In the present work, the so-called added dissipation zone method of Chiba and $\mathrm{Ku}$ wahara (cf. Hinatsu ${ }^{27}$ ) is used. In this method the computational domain is extended with a coarse grid, which induces a numerical dissipation effect, and waves are damped by this sort of numerical damping. A Neumann-type boundary condition is imposed on the outmost boundary. The coarse grid is obtained by increasing the grid size toward open boundaries with a geometrical progression. A fictitious damping force was included in the $\xi^{2}$ direction in the added dissipation zone by Hinatsu, ${ }^{27}$ but it is found unnecessary in the present work.

The initial velocity field is taken to be that of the static (quiescent) fluid case, that is, $u_{1}=u_{2}=0$, and a constant acceleration is imposed in the entire domain until the inflow velocity attains one.

\section{DISCRETIZATION AND SOLUTION PROCEDURE}

A regular grid is used in the present method for discretization. The velocity and pressure are evaluated at computational cell centers. The advantage of this stencil is that the conservation of mass and momentum are exactly satisfied, even in the discretized formulation. Spatial differentials are discretized using the second-order central difference, and the QUICK scheme of Leonard ${ }^{30}$ is used for discretization of convection terms. Time marching is carried out using a timesplitting fractional-step method. It is a two-step predictorcorrector scheme. In the predictor step, an intermediate velocity field $\tilde{u}_{i}$ is computed explicitly by integrating Eq. (5) in time using the velocity and pressure from previous time step $n$,

$$
\begin{aligned}
\frac{\tilde{u}_{i}}{J^{n+1}}-\left(\frac{u_{i}}{J}\right)^{n}= & \Delta t\left[-\frac{\partial}{\partial \xi^{j}}\left(\frac{u_{i}}{J} U^{j}\right)-\frac{\partial}{\partial \xi^{j}}\left(\frac{\phi}{J} \frac{\partial \xi^{j}}{\partial x_{i}}\right)\right. \\
& \left.+\frac{1}{\operatorname{Re}} \frac{\partial}{\partial \xi^{j}}\left(\frac{1}{J} g^{j l} \frac{\partial u_{i}}{\partial \xi^{l}}\right)\right]^{n},
\end{aligned}
$$

where $\Delta t$ is the time increment and the superscripts $n$ and $n+1$ denote the previous and the present time steps, respectively. After the predictor step, the location of the free surface at time step $n+1$ is evaluated by integrating Eq. (13) explicitly, 


$$
h^{n+1}=h^{n}+\Delta t\left(u_{2}-u_{1} \frac{\partial \xi^{i}}{\partial x_{1}} \frac{\partial h}{\partial \xi^{i}}\right)^{n},
$$

and the grid of the flow domain is regenerated to fit the newly estimated free surface. The Jacobian and the metric tensor are recalculated according to the new grid.

At the second step, $\tilde{u}_{i}$ is corrected by the pressure increment $\delta \phi=\phi^{n+1}-\phi^{n}$ between the present time step $n+1$ and the previous time step $n$ to obtain the velocity field $u_{i}^{n+1}$ at the present time step $n+1$,

$$
\begin{aligned}
\left(\frac{u_{i}}{J}\right)^{n+1}-\frac{\tilde{u}_{i}}{J^{n+1}}= & -\Delta t\left[\frac{\partial}{\partial \xi^{j}}\left(\frac{\phi}{J} \frac{\partial \xi^{j}}{\partial x_{i}}\right)\right]^{n+1} \\
& +\Delta t\left[\frac{\partial}{\partial \xi^{j}}\left(\frac{\phi}{J} \frac{\partial \xi^{j}}{\partial x_{i}}\right)\right]^{n} \\
\cong & -\Delta t\left[\frac{\partial}{\partial \xi^{j}}\left(\frac{\delta \phi}{J} \frac{\partial \xi^{j}}{\partial x_{i}}\right)\right]^{n+1} .
\end{aligned}
$$

Note that the difference of the pressure differential between $n+1$ and $n$ time steps is simply approximated by the differential of the pressure increment at the $n+1$ time step. However, the pressure increment $\delta \phi$ in Eq. (20) is not known and must be determined by requiring the velocity field $u_{i}^{n+1}$ to satisfy the continuity equation. The divergence-free condition applied for $u_{i}^{n+1}$ yields a Poisson equation for $\delta \phi$ by taking the divergence of Eq. (20). Thus

$$
\left[\frac{\partial}{\partial \xi^{i}}\left(\frac{\tilde{U}^{i}}{J}\right)\right]^{n+1}=\Delta t\left\{\frac{\partial}{\partial \xi^{k}}\left[\frac{\partial \xi^{i}}{\partial x_{k}} \frac{\partial}{\partial \xi^{j}}\left(\frac{\delta \phi}{J} \frac{\partial \xi^{j}}{\partial x_{i}}\right)\right]\right\}^{n+1},
$$

where $\tilde{U}^{i}=\left(\partial \xi^{i} / \partial x_{j}\right) \tilde{u}_{j}$.

The unsteady problem is treated as a quasisteady one between successive physical time steps. The known value of a dependent variable at time $t$ can be used as a guess for the unknown value of that variable at time $t+\Delta t$ for reasonably small $\Delta t$. Since this is a relatively good guess, only a few iterations are normally needed to obtain a converged solution for time $t+\Delta t$. The grid is aligned to the free-surface boundary, which moves in time. Therefore the grid is time dependent in general. However, since Eq. (5) is integrated in time by the explicit method, within each time step the grid is independent of time (cf. Farmer et al. ${ }^{28}$ ). Therefore the grid point speed terms, $\partial \xi^{j} / \partial t$, are dropped in Eqs. (18) and (19).

In summary, if the location of the free surface and the values of the velocity and pressure in the calculation domain are known at time step $n$, the solution procedure for the next time step $n+1$ is as follows.

(1) Compute the intermediate velocity $\tilde{u}_{i}$ explicitly by solving the momentum equation (18), using the velocity and pressure from time step $n$.

(2) Determine the new location of the free surface at time step $n+1$ by the kinematic free-surface boundary condition, Eq. (19), and then regrid the flow domain to fit with the new location of the free surface. Jacobian and metric tensor are recalculated according to the new grid.

(3) Solve the Poisson equation (21) implicitly to compute the pressure increment $\delta \phi$ between the time steps $n$ and $n+1$, then update the pressure by $\phi^{n+1}=\phi^{n}+\delta \phi$.
(4) Correct the intermediate velocity field $\tilde{u}_{i}$ by Eq. (20) using the pressure increment $\delta \phi$ estimated in step (3) to obtain the velocity field $u_{i}^{n+1}$ at time step $n+1$.

(5) Obtain the velocity component $u_{1}$ on the free surface by extrapolation from the interior using the velocity gradient $\partial u_{1} / \partial \xi^{2}$ on the free surface obtained by solving Eqs. (10) and (11) simultaneously. Calculate $u_{2}$ on the free surface from the continuity equation (2).

(6) Return to step 1 for the next time step.

\section{VALIDATION AND DISCUSSION}

The viscous free-surface flow studied experimentally and numerically by Lee $e t a l{ }^{8}$ is used to validate the present computational method. The experiments of Lee $e t$ al. ${ }^{8}$ were conducted in an open flume of $7.5 \mathrm{~m}$ long, $0.75 \mathrm{~m}$ wide, and $0.6 \mathrm{~m}$ deep. The bottom unevenness was $\pm 0.1 \mathrm{~cm}$. A twodimensional body of the arched cross section with a chord of $4.9 \mathrm{~cm}$, height of $0.65 \mathrm{~cm}$ at its midchord, and span of $72 \mathrm{~cm}$ was used. It was positioned just above the floor of the flume with a gap of $0.05 \mathrm{~cm}$ at the highest location of the floor. Because of the unevenness of the floor, the gap between the base of the body and the floor of the tank varied from $0.0125 h_{0}$ to $0.0625 h_{0}$, with an average value of $0.0375 h_{0}$. Two water depths, i.e., 4.0 and $5.33 \mathrm{~cm}$, were used for measurements over a range of Froude number from 0.7 to 1.1. To eliminate any sidewall effects the wave elevations were measured at points as close as possible to the longitudinal centerplane of the tank. Lee et al. ${ }^{8}$ estimated the effects of viscosity for the attenuation of a solitary wave in a channel with smooth sidewalls using the empirical formula suggested by Daily and Stephan. The estimation indicated that a soliton would attenuate less than 5\% at the end of the experiments. Thus, the experiments could be considered as two dimensional and the effects of viscosity on wave propagation from smooth sidewalls were not corrected for in the comparison between experimental data and numerical results from twodimensional computations given below.

\section{A. Numerical simulation}

The exact geometry of the body used in the experiments with an average gap of $0.0375 h_{0}$ between the base of the body and the channel bottom is used for the present numerical simulation. The length of the fluid domain is taken as 100 units, and the depth of water is set to be unity. 232 grid points are used in the horizontal direction, and 31 grid points are given in the vertical direction. The grids are constructed in such a way that the $\xi^{1}=$ const grid lines are all vertical, i.e., $\xi^{1}$ is a function of $x_{1}$ only. The grid lines are concentrated around the body, near free surface, and the floor of the tank. A partial view of the initial grids for numerical computation is shown in Fig. 2. The grid system is rearranged at each time step to fit the deformed free surface.

The time step is set to be $\Delta t=0.01$. The computation is started at the initial condition of zero velocity and pressure everywhere and a flat free surface $(h=1.0)$. A constant acceleration of 0.25 , or $0.25 \mathrm{~g}$ in the dimensional space, is imposed on the entire domain until the inflow velocity reaches unity. For the time step of $\Delta t=0.01,400$ time steps are needed to accelerate the flow from zero velocity until the 


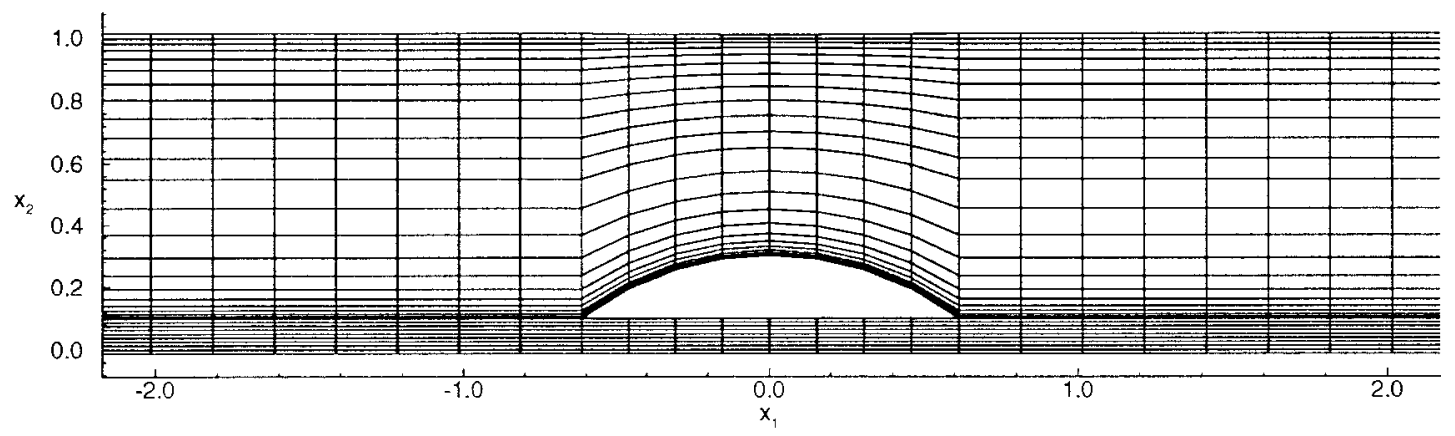

FIG. 2. A partial view of the numerical grids.

upstream inflow velocity reaches unity. Since the acceleration in the experiments was uncertain, the acceleration for the present numerical computation is determined by numerical tests. The calculation is carried out for two water depths, i.e., $h_{0}=4.0 \mathrm{~cm}$ and $h_{0}=5.33 \mathrm{~cm}$, and at three Froude numbers, i.e. subcritical, transcritical, and supercritical, for each water depth corresponding to those in the experiments. All other parameters in the calculation are the same as those in the experiments in order to make a meaningful comparison. In the experiments, wave gauges were fixed in the channel and located at 70 and 68.1 units upstream of the body for the water depth $h_{0}=4.0 \mathrm{~cm}$ and $h_{0}=5.33 \mathrm{~cm}$, respectively. In the calculation with a body frame reference, a numerical wave gauge is initially located at the same distance upstream of the body as it was in the experiments, and then moves steadily with the free-stream velocity.

\section{B. Comparison with experimental data}

The results of the present calculation along with the experimental data and the numerical solutions predicted using the g-B model and the fKdV model by Lee et al. ${ }^{8}$ are shown in Fig. 3 for $h_{0}=4.0 \mathrm{~cm}$ at $F n=0.89,1.01,1.12$, and in Fig. 4 for $h_{0}=5.33 \mathrm{~cm}$ at $F n=0.90,1.01,1.11$, respectively. The plots at the top of Fig. 3 and Fig. 4 are from the present numerical results, which show the surface elevation $h$ as a function of $x_{1}\left(x_{1}=x\right)$ and $t$ at $F_{n}=1.01$ for $h_{0}=4.0 \mathrm{~cm}$ and $h_{0}=5.33 \mathrm{~cm}$, respectively. As can be seen from the plots, the present NS solutions predict all the main features of the phenomena indicated earlier, namely the upstream-advancing solitary waves, the prolonging depressed region immediately behind the disturbance, and the train of trailing waves. These features were observed in the experiments.

Detailed comparisons for the free-surface elevation as a function of time recorded by wave gauge are made in the rest of the plots in Fig. 3 and Fig. 4. Overall, it is seen that the present NS solutions give excellent agreement with the experiments. The amplitude, phase, and number of solitary waves are very well predicted for all three Froude numbers in both water depths. For the trailing waves the agreement between the present NS solutions and the experiments is considered to be good, except for the case of the relatively strong disturbance $\left(h_{0}=4.0 \mathrm{~cm}\right)$ at subcritical speed, $F n$ $=0.89$ (Fig. 3), where variance between the numerical and experimental data is most pronounced. According to Lee et al. ${ }^{8}$ it is precisely in this case that the wave breaking was observed during the experiments. Since the kinematic condition is implemented by the Eulerian method in the present numerical scheme, it only permits solutions in which the free-surface location $h$ is single valued. Consequently, it does not allow for the breaking of waves.

\section{Comparison with the $\mathrm{KKdV}$ and the $\mathrm{g}-\mathrm{B}$ models}

Lee et $a l .{ }^{8}$ presented numerical results corresponding to the experimental conditions using two inviscid-flow models, i.e., the fKdV model and the g-B model. In an attempt to account for the bottom unevenness, the bottom-bump clearance, and the presence of a viscous boundary layer along the channel floor and on the body surface, an effective bump height of $0.8 \mathrm{~cm}$ was assumed by Lee et al. ${ }^{8}$ instead of the actual bump height of $0.65 \mathrm{~cm}$. The numerical results of the fKdV and g-B models are also shown in Fig. 3 and Fig. 4 for comparison. It can be seen from the figures that the fKdV model predicts a phase lead of the upstream-advancing solitary waves for the strong disturbance $\left(h_{0}=4.0 \mathrm{~cm}\right)$ case at subcritical and critical speeds, and underpredicts the upstream-advancing waves at all three Froude numbers for the weak disturbance $\left(h_{0}=5.33 \mathrm{~cm}\right)$ case. For the trailing waves the fKdV model overpredicts the amplitude. The g-B model predicts a phase lag of the upstream-advancing solitary waves and a phase lead of the trailing waves for all the cases, and underpredicts the amplitude and number of the upstream-advancing waves for the weak disturbance case. Both fKdV and g-B models fail to predict the trailing waves for the relatively strong disturbance $\left(h_{0}=4.0 \mathrm{~cm}\right)$ case at subcritical speed, $F n=0.89$, where wave breaking was observed in the experiments.

Throughout these comparisons it reveals that the present NS method performs better than the two simplified inviscidflow models. The improved agreement with experiments by the NS solution may be due to the following reasons. First, the fKdV and g-B models cannot simulate the detailed flows around a submerged body because they are depth-averaged models, whereas the NS equations do not have such restrictions. Second, the geometry of the body used in the numerical solutions of the fKdV and the g-B models is different from that in the experiments by an effective bump height instead of the actual bump height. Third, the presence of a viscous boundary layer around the body may be thought to increase the effective disturbance caused by the body. And furthermore, a Couette flow develops in the gap region be- 

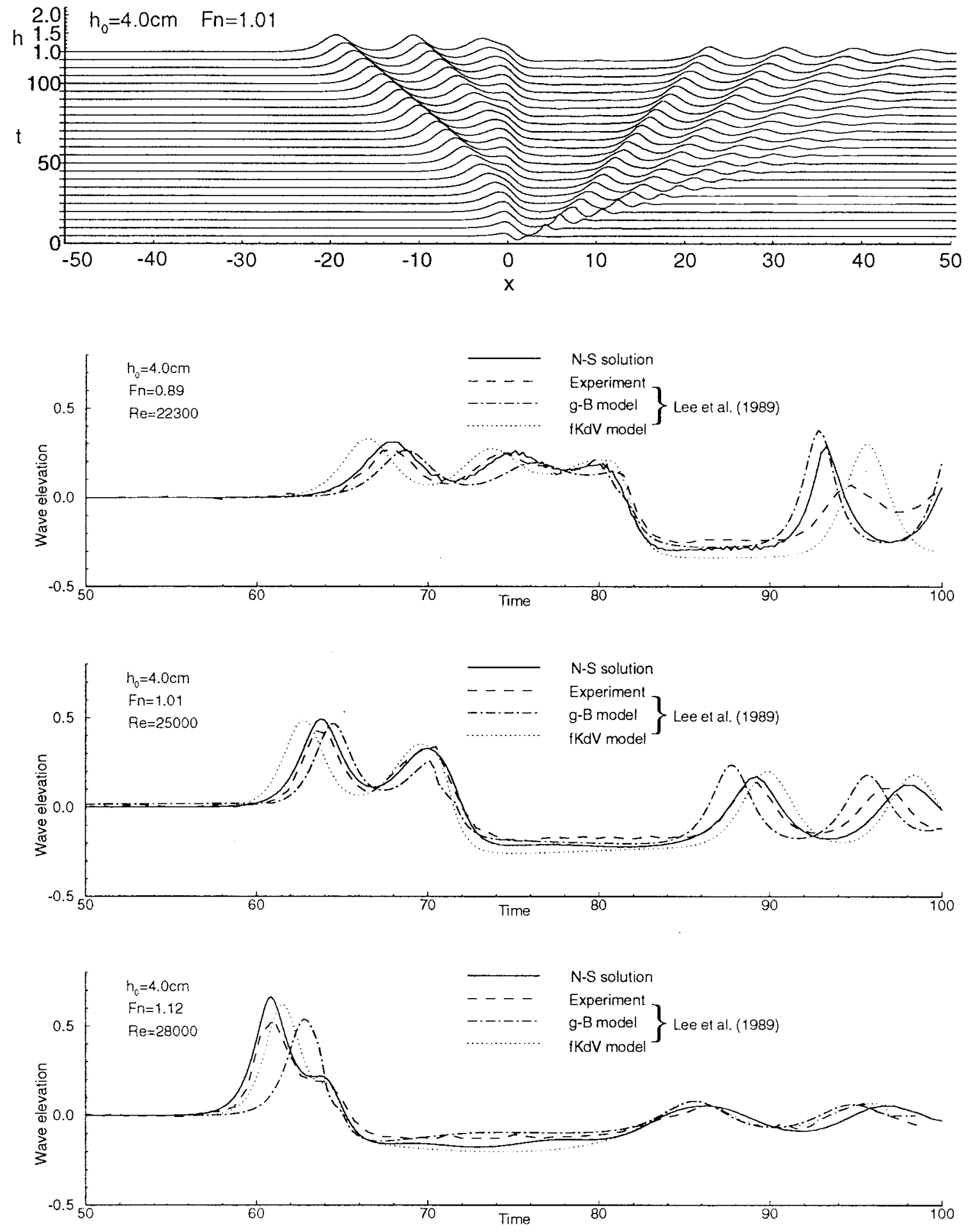

FIG. 3. Comparison between experimental data and numerical results for water depth $h_{0}=4.0 \mathrm{~cm}$ at various Froude numbers.

tween the body and the channel bottom, which in turn induces some back-pressure gradient and impedance to the flow through the clearance. In the calculation of the inviscid fKdV and g-B models, these viscous effects are simply accounted for by increasing the bump height, i.e., increasing the strength of the disturbance. On the other hand, in the solutions of the NS equations, viscosity is included in the modeling. Therefore, the viscous boundary layer around the body and Couette flow in the small gap can be accurately simulated by the NS equations. In order to investigate the effect of the viscous boundary layer within the gap on the free-surface waves, a calculation of the NS equations for a case in which the body touches the bottom of the channel (without a gap) is conducted. The results reveal that the waves generated on the free surface without a gap (where the body touches the bottom of the channel) are quite different 

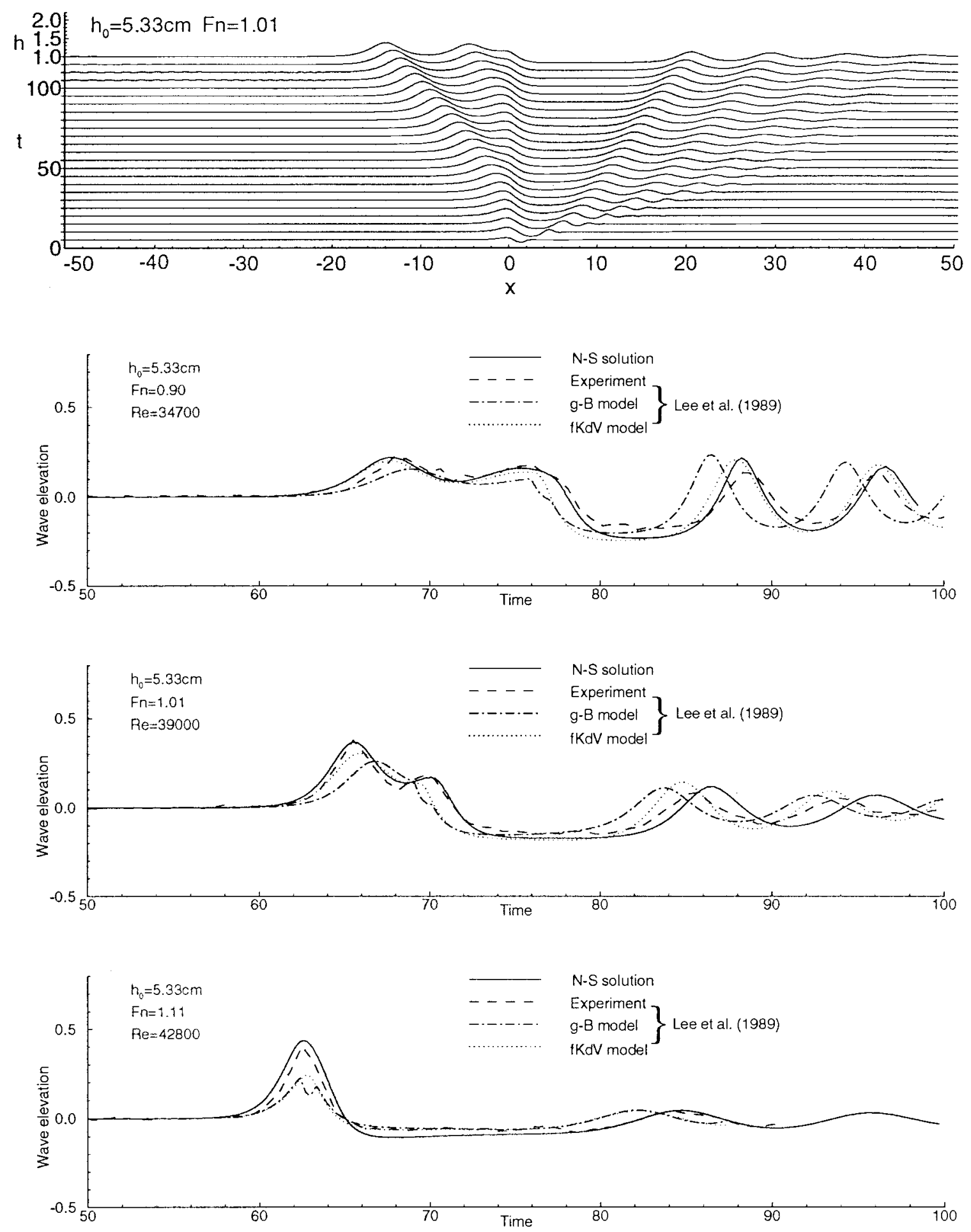

FIG. 4. Comparison between experimental data and numerical results for water depth $h_{0}=5.33 \mathrm{~cm}$ at various Froude numbers.

from those with a gap, and the former does not agree with the experimental data (see Fig. 5). Small-amplitude advancing solitary waves with low phase velocity are observed for the case where the body touches the channel bottom. This indicates that the viscous effect in the boundary layer around the body and on the floor of the tank plays an important role in the generation of solitary waves. It is interesting to note that the results given by the inviscid-flow models, such as the
fKdV and g-B models, are independent of the gap size and their accuracy depends, to a great extent, on the accuracy of estimating the viscous effect around the body and on the channel floor, and on the way of approximately accounting for it.

The Reynolds numbers used for the present NS solutions are the same as those in the experiments of Lee et al. ${ }^{8}$ which are of the order of $10^{5}$. At sufficiently high Reynolds num- 


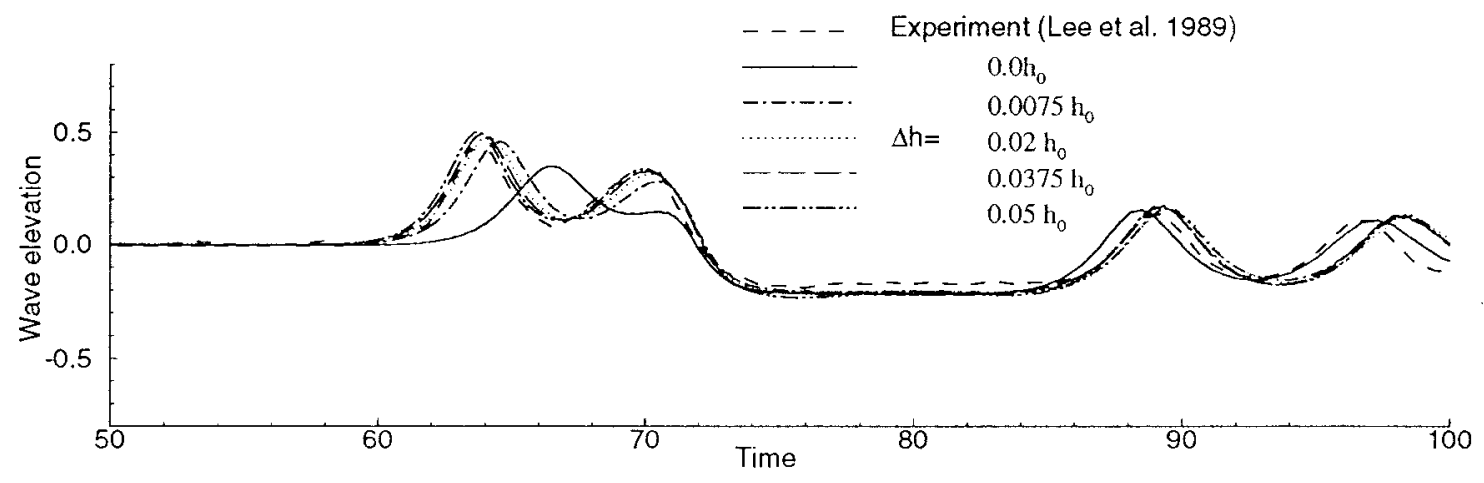

FIG. 5. Wave profiles for different clearance $(\Delta h)$ between the base of a submerged body and the channel bottom.

bers, the vorticity is confined to a thin boundary layer along the free surface. The source of vorticity at the bottom of the channel and on the boundary of a submerged body is much stronger than that on the free surface (cf. Tang et al. ${ }^{24}$ ). Therefore the improved agreement with experiments by the NS solutions is primarily due to the fact that the viscous effect in the boundary layer around the body and on the floor of the channel can be correctly simulated in the NS equations. Unfortunately no equivalent calculation of full Laplace equation is available for comparison with the experiments of Lee et $a .^{8}$ and with the present NS solutions. However, according to Cao and Beck, ${ }^{20}$ the numerical results from the fully nonlinear model (with the complete Laplace field equation and fully nonlinear free surface boundary conditions) agree with the results of the fKdV model for a weak surface pressure disturbance, while the $\mathrm{fKdV}$ equation predicts no difference in waves generated by two different types of disturbances of the same distribution, i.e. the bottom topography and the surface pressure.

\section{VARIATION OF THE SUBMERGENCE DEPTH}

In the experiments of Lee et al.,$^{8}$ a series of tests were conducted to determine if the vertical position of the body in a water column would influence the resultant wave development. It was reported that the waves generated were quite independent of the disturbance position.

Both the fKdV model and the g-B model admit forcing functions in the form of a surface pressure distribution or a bottom topography. For the fKdV model, these two forcing functions are entirely equivalent, whereas for the g-B model there exists a very mild difference between them. This suggests that a surface pressure would produce a quite similar solution in comparison to a bottom topography of the same distribution. Both models were derived by assuming that the $x$-component velocity of the fluid was a water-layer depthaveraged velocity, and its dependence on the vertical dimension was removed. In practical applications, this means that the resultant motion induced by a horizontally moving thin body would not depend on its submergence depth. Thus, both models cannot be used to calculate waves generated by an arbitrarily submerged body. However, the NS equations can be used to calculate the waves generated by either a bottom topography, a submerged body, or a free-surface disturbance.

A series of calculation is conducted with the present NS method for the vertical position of a body, varying from the bottom of the channel to the middle of the water depth. Figure 6 shows the variation of the amplitude of the first upstream-advancing solitary wave, with the clearance between the base of a submerged body and the channel bottom. It can be seen that the wave amplitude has a steep increase versus the clearance from zero to a very small value (about $\left.0.0075 h_{0}\right)$. It then slowly increases with the clearance until the clearance is about $0.05 h_{0}$, beyond that the wave amplitude remains almost unchanged.

The big difference of the wave amplitude between zero clearance and a small clearance is due to the viscous flow induced in the gap region, which causes a sudden increase in the wave amplitude. The presence of a viscous boundary layer on the base of the body and on the channel floor in the gap region may be thought to increase the effective strength of the body. The viscous effect increases slowly with increasing clearance as the extension of the viscous boundary layer in the gap region. Up to a certain stage (the clearance is about $0.05 h_{0}$ in the present case), the viscous flow is fully developed in the gap. A further increase in clearance would not increase the viscous effect. This supports the theory and agrees with the observation of experiments that the wave generated by a submerged body would not depend on its submergence depth, except for a very small region close to the bottom of the channel.

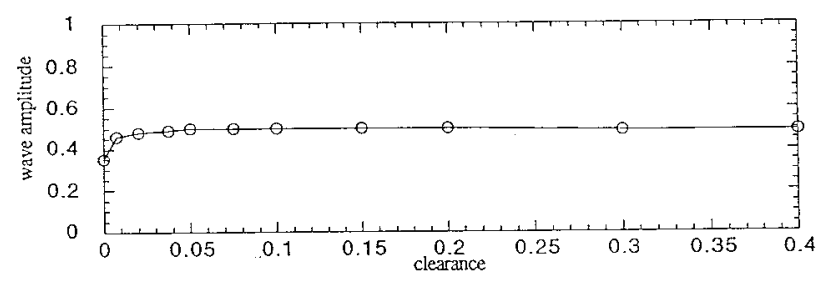

FIG. 6. Variation of the amplitude of the first upstream-advancing solitary wave with the clearance between the base of a submerged body and the channel bottom. 


\section{CONCLUSIONS}

The present numerical method of solving the unsteady, two-dimensional, Navier-Stokes equations for free-surface flows predicts the main features of the solitary waves generated by a submerged disturbance moving steadily at a speed near the critical velocity in a shallow water channel. The numerical results from the present method agree very well with the experimental data, and the present method performs better than the simplified potential-flow models, i.e., the g-B model and the fKdV model. The improved agreement with experiments by the present method is primarily due to the fact that the viscous effect in the boundary layer around the body and on the channel floor can be correctly simulated in the computation of the NS equations. The present numerical calculation shows that the vertical position of a submerged body would not influence the resultant wave development, except for a very small region close to the bottom of the channel.

\section{ACKNOWLEDGMENTS}

The authors are grateful to Dr. M. Hinatsu for his comments on the computational method and to Dr. G. T. Yates for valuable discussion. This research was sponsored by the University of Hong Kong, under CRCG Grant Nos. 337/064/ 0025 and 337/064/0032.

${ }^{1}$ J. G. Thews and L. Landweber, "The influence of shallow water on the resistance of a cruiser model," U.S. Experimental Model Basin Report 408, Washington, DC, Navy Yard, 1935.

${ }^{2}$ D. B. Huang, O. J. Sibul, W. C. Webster, J. V. Wehausen, D. M. Wu, and T. Y. Wu, "Ships moving in the transcritical range," in Proceedings of the Conference on Behaviour of Ships in Restricted Waters, Varna, Bulgarian Ship Hydrodynamics Centre, 1982, Vol. II, pp. 26-1-26-10.

${ }^{3}$ R. C. Ertekin, W. C. Webster, and J. V. Wehausen, "Ship-generated solitons," Proceedings of the 15th Symposium on Naval Hydrodynamics, Hamburg, 1984, pp. 347-364.

${ }^{4}$ M. G. Sun, "The evolution of waves created by a ship in shallow canal," in the 60th Anniversary Volume, Zhongshan University, Mechanics Essays Guangzhow, China, 1985, pp. 17-25 (in Chinese).

${ }^{5} \mathrm{D}$. M. Wu and T. Y. Wu, "Three-dimensional nonlinear long waves due to moving surface pressure," in Proceedings of the 14th Symposium on Naval Hydrodynamics, Washington DC, 1982, pp. 103-125.

${ }^{6}$ T. Y. Wu, "Long waves in ocean and coastal waters," J. Eng. Mech. Div. ASCE 107, 501 (1981).

${ }^{7}$ T. Y. Wu, "Generation of upstream advancing solitons by moving disturbances," J. Fluid Mech. 184, 75 (1987).

${ }^{8}$ S. J. Lee, G. T. Yates, and T. Y. Wu, "Experiments and analyses of upstream-advancing solitary waves generated by moving disturbances," J. Fluid Mech. 199, 569 (1989).

${ }^{9}$ T. R. Akylas, "On the excitation of long nonlinear water waves by a moving pressure distribution,” J. Fluid Mech. 141, 455 (1984).
${ }^{10}$ S. L. Cole, "Transient waves produced by flow past a bump," Wave Motion 7, 579 (1985).

${ }^{11}$ R. H. J. Grimshaw and N. Smyth, "Resonant flow of a stratified fluid over topography," J. Fluid. Mech. 169, 429 (1986).

${ }^{12}$ C. C. Mei, "Radiation of solitons by slender bodies advancing in a shallow channel," J. Fluid Mech. 162, 53 (1986).

${ }^{13}$ R. C. Ertekin, W. C. Webster, and J. V. Wehausen, "Waves caused by a moving disturbance in a shallow channel of finite depth," J. Fluid Mech. 169, 275 (1986)

${ }^{14} \mathrm{D}$. M. Wu and T. Y. Wu, "Precursor solitons generated by threedimensional disturbances moving in a channel," in Nonlinear Water Waves, IUTAM Symposium, Tokyo, Japan, 1987, pp. 69-75.

${ }^{15}$ C. Katsis and T. R. Akylas, "On the excitation of long nonlinear water waves by a moving pressure distribution. Part 2 . Three-dimensional effects," J. Fluid Mech. 177, 49 (1987).

${ }^{16}$ M. S. Longuet-Higgins and E. D. Cokelet, "The deformation of steep surface waves on water, I. A numerical method of computation," Proc. R. Soc. London Ser. A 350, 1 (1976).

${ }^{17} \mathrm{~K}$. Suzuki, "Calculation of nonlinear water waves around a 2-dimensional body in uniform flow by means of boundary element method," 5 th International Conference on Numerical Ship Hydrodynamics, Hiroshima, Japan, 1989, pp. 157-167.

${ }^{18}$ R. Cointe, "Nonlinear simulation of transient free surface flows," in Ref. 17, pp. $168-179$.

${ }^{19} \mathrm{R}$. W. Yeung and M. Vaidhyanathan, "Nonlinear interaction of water waves with submerged obstacles," Int. J. Num. Methods Fluids 14, 1111 (1992).

${ }^{20}$ Y. Cao and R. F. Beck, "Numerical computations of two-dimensional solitary waves generated by moving disturbances," Int. J. Num. Methods Fluids 17, 905 (1993).

${ }^{21}$ F. H. Harlow and J. E. Welch, "Numerical calculation of time-dependent viscous incompressible flow of fluid with free surface," Phys. Fluids 8 , 2182 (1965)

${ }^{22}$ R. K. C. Chan and R. L. Street, "A computer study of finite-amplitude water waves,” J. Comput. Phys. 6, 68 (1970).

${ }^{23} \mathrm{H}$. Miyata and S. Nishimura, "Finite-difference simulation of nonlinear ship waves," J. Fluid Mech. 157, 327 (1985).

${ }^{24}$ C. J. Tang, V. C. Patel, and L. Landweber, "Viscous effects on propagation and reflection of solitary waves in shallow channels," J. Comput. Phys. 88, 86 (1990).

${ }^{25} \mathrm{R}$. W. Yeung and P. Ananthakrishnan, "Oscillation of a floating body in a viscous fluid," J. Eng. Math. 26, 211 (1992).

${ }^{26}$ J. H. Chang and C. J. Tang, "Viscous effects on nonlinear water waves generated by a submerged body in critical motion," Proceedings of the 17th National Conference on Theoretical and Applied Mechanics, Taipei, Taiwan, 1993, pp. 35-42.

${ }^{27}$ M. Hinatsu, "Numerical simulation of unsteady viscous nonlinear waves using moving grid system fitted on a free surface," J. Kansai Soc. N.A. Jpn. 217, 1 (1992)

${ }^{28}$ J. Farmer, L. Martinelli, and A. Jameson, "Fast multigrid method for solving incompressible hydrodynamic problems with free surfaces," AIAA J. 32, 1175 (1994).

${ }^{29}$ S. Y. Boo and C. H. Kim, "Simulation of fully nonlinear irregular waves in a 3-D numerical wave tank," Proceedings of the 4th International Offshore and Polar Engineering Conference, Osaka, 1994, pp. 17-24.

${ }^{30}$ B. P. Leonard, "A stable and accurate convective modelling procedure based on quadratic upstream interpolation," Comput. Methods Appl. Mech. Eng. 19, 59 (1979). 